\title{
The Adoption of Software Process Improvement in Saudi Arabian Small and Medium Size Software Organizations: An Exploratory Study
}

\author{
Mohammed Ateeq Alanezi \\ College of Computing and IT \\ Shaqra University, Kingdom of Saudi Arabia
}

\begin{abstract}
Quite a lot of attention has been paid in the literature on "how to adopt" software process improvement (SPI) in Small and Medium Size (SME) software organization in several countries. This has resulted in limited improvements to the software industry and impacted the Saudi's economy. However, the SPI adoption is one of the major issues in the domain of small and medium size software organization, especially in developing countries. The objective of this study is to investigate the current state of SPI adoption in Saudi Arabia in comparison to those of the standard models used internationally which could help in improving the software quality and have an impact on Saudi Arabian economy. After examining a number of studies in the literature, we have designed a questionnaire to survey SME software organizations in Saudi Arabia. First, we conducted a pilot study with 24 senior managers to access the intended survey and further improve the process. Then, we sent out 480 questionnaires to the participants and received 291 responses. The most interesting part of this result is that the respondents highlighted the benefits of using SPI standard; whereby, when asked about the reason for not using SPI, $64 \%$ of the respondents agree that the usage of SPI standard is time consuming and $55 \%$ agree that there is a difficulty in understanding the SPI standard.
\end{abstract}

Keywords-Software process improvement; software organization; software adoption; small and medium enterprises (SME)

\section{INTRODUCTION}

Saudi Arabia is developing rapidly in the field of information technology. The number of software organizations in Saudi Arabia is growing daily. Within these organizations, many activities, tasks, scheduling and resources need to be managed and monitored properly by the respective development team. However, according to [1], in the past 5 years, clients have faced difficulties in getting high quality products which are reliable and cheap. This is somewhat considered reasonable since the software industry is relatively young in Saudi Arabia. Accordingly, the challenge for Saudi Arabia software organizations is to find a path to apply Software Process Improvement (SPI) techniques to achieve high quality process. The motivation behind SPI, usually, come as a result of business needs such as strong competition, reduced schedule cycle times, increased product quality and hence more productivity and profit [1], [2]. Several SPI standards and models have been proposed to control software development processes, such as the Capability Maturity Model
Integration (CMMI) [3], International Organization for Standardization ISO 9000 [4], the Software Process Improvement and Capability determination (SPICE) [5], [6], Bootstrap [7], [8], Six Sigma [9]-[11]. However, despite the importance of SPI, it is not clear how these practices are implemented and whether the required skills and knowledge are owned by practitioners. In addition, many studies have proved that all of these models are very difficult to apply in small and medium size software organizations [12]-[16]. Nevertheless, most of the software organizations in the world are considered as small-to-medium size; for example, small-tomedium size organizations represent 97.3 of the total business established in Malaysia [17], whereas they represents $92 \%$ in Mexico [18] and more than $85 \%$ in India, Canada, China, US, Ireland and Finland [19]. A number of studies have investigated the adoption of SPI models in several countries, for example, in India [19], Malaysia [17], [20]-[23], US and Japan [24], New Zealand [25], Finland [26], Pakistan [27], Australia [23], [24] Ireland [30] and Mexico [18]. However, to the best of our knowledge, no prior work exists which aims at specifically investigating the adoption of SPI in Saudi Arabia. Hence, it is significant to investigate the current state of the SPI adoption to help improve the software industry and its impact on the Saudi Arabian economy. We are not going to measure the extent to which the improved processes have been fully adopted, or if the processes have really changed the hearts and the minds of the practitioners. However, this research attempts to explore, analyze, and evaluate the adoption of SPI in Saudi Arabian software organizations, and then understand the current perspective of software process in comparison to those of the standard models used internationally. More specifically, we aim to answer the following questions:

- What is the state of SPI adoption in Saudi Arabia?

- What are the factors that influence the usage of SPI Standard and the reasons behind not using the SPI standards? SPI is significant because in primary it means a new and enhanced software development process is created.

- Have the employees had a clear explicit understating of the SPI theory? This is because it is difficult to apply the SPI model without fully understanding the model theory behind it. 
- To what extent can the process activity be supported by Computer Aided Software Engineering tool.

This paper is organized as follows: Section II discusses related works, Section III describes the methodology used in the study, Section IV presents the results of the study as well as our interpretation of these findings, Section V presents discussion of the results and finally, the paper is concluded in Section VI.

\section{RELATED WORK}

Several published researches were conducted to investigate the adoption of SPI in different parts of the world. A survey of six small-to-medium size software organizations investigated the adoption of SPI in Malaysian [16]. The results of the study showed that the level of adoption of SPI in Malaysia is still very much at the low level. Nizam et al. [31] also surveyed 39 organizations which operate in Malaysia to analyze factors that negatively influence the adoption of SPI. They concluded that the adoption of SPI is still at its early stage in small and medium size organizations. This shows that Malaysian software organizations are mostly not aware of the importance of SPI and its impact on product quality. Another survey on SPI implementation with 50 small-to-medium Indian companies is conducted in [19]. The findings showed that developers were responding relatively positively to SPI. Similarly, 15 companies showed their eagerness for achieving a CMMI level as the primary goal. In recent study, Mahmood Niazi [28] investigated the risks that can undermine SPI implementation from the perspective of software development practitioners. He interviewed 34 Australian's SPI practitioners, and the results clearly identified the differences and the similarities of the risks by organizational size (i.e. smallmedium and large) and practitioner's positions (i.e. developers, managers and senior managers). However, the small sample data and the week methodology are not sufficient to draw statistically significant conclusions. The reasons why organizations do not adopt CMMI in Australian companies were investigated in [29]. The outcomes showed the most frequent reasons given by organizations were: 1) the organization was small; 2) the adoption was too costly, and 3) the organization had no time. In [16] a survey was conducted over 400 volunteers from 32 countries to question small organizations about their utilization of SPI models. The results showed that many difficulties must be overcome to consider the process effectiveness. As we have seen in the above literature, there were numerous publications which study the adoption and implantation of SPI models in Malaysia, India, Pakistan, Australia, New Zealand, Mexico and United States. Notably, there is still a huge gap of research and published studies on the adoption of SPI in Saudi Arabian.

\section{RESEARCh DESIGN AND METHOD}

Since this is an exploratory research, the author wanted to get both a broad view of the software organization in Saudi Arabia as a whole, and a more detailed picture of SPI practices. Initially, a detailed literature review on the adoption of SPI model was performed, looking at the implementation of SPI in context of SME organizations, the key success factors and the difficulties of the adoption of SPI. After that, a questionnaire was created to investigate the adoption of SPI in the country's SMEs. Questionnaire approach have been used in a number of similar studies and are presented as a proven technique of data gathering and analysis [17], [19], [25], [27]. In this section, the details of the survey are given as shown in the following subsections:

\section{A. Survey Design}

A number of experienced studies were analyzed in order to identify questions that can play a positive role in the adoption of SPI model. The questionnaire consisted of 21 questions that were selected mainly from [17], [19], [20], [31], [32]. A pilot study with 24 senior managers was conducted to validate the questionnaire. After considering their comments, the questionnaire was modified and improved. All questions were close ended in order to get better number of respondents. The questionnaire consists of four major parts to capture the required data as follows:

- The first part is background information of the companies (size, status, market access, and culture).

- The second part aims to get information about the respondents (education, experience, and job nature).

- The third part contains SPI practices and knowledge, and the last part concerns about software development practice and related project issues.

To conduct this survey, Google forms which is a websurvey tool was adopted.

\section{B. Survey Approach}

The empirical method used in this study is survey approach. This approach is properly common used method to collect data from targeted respondents who have the required knowledge to address the objective of the study.480 SMEs organizations were identified to be suitable candidates for the survey through the small and medium enterprises general authority's web site ${ }^{1}$. Then, a short letter that explains the research objectives along with the questionnaire was sent to them and 291 responses were received. The response rate was acceptable due to the privacy policies of the organizations. The duration of questionnaire was four weeks along with follow up emails to resolve raised confusions.

\section{Analysis}

Two basic statistical methods were used to analyze the collected quantitative data; the methods are descriptive statistics and frequent analysis. Additionally, the results were revised by two experts separately to guarantee the accuracy of the outcome.

\section{RESULTS}

This section presents the analysis performed on the information gathered from the survey. It shows that a welldesigned development process has positive consequences on the productivity and cost. On the negative side, poor software

\footnotetext{
${ }^{1}$ https://smea.gov.sa/en
} 
development has negative impact on the quality and poor customer satisfaction [33].

Respondents were asked about their opinion on the factors which influence their usage of SPI Standard and the reasons for not using the SPI standards. To simplify these two questions, a list of some factors affecting the usage of SPI standard were selected carefully from the literature [17], [19], [20], [31], [32]. First, an overview of the respondents' impression of the benefits of using the SPI standards is presented and it is shown in Fig. 1. In this 3D graph, the $\mathrm{X}$-axis (horizontal line) present the benefits of using SPI standard, the y-axis (left side of the graph) presents the number of respondents and on the $\mathrm{z}$-axis (right side of the graph), the rating scales are shown.

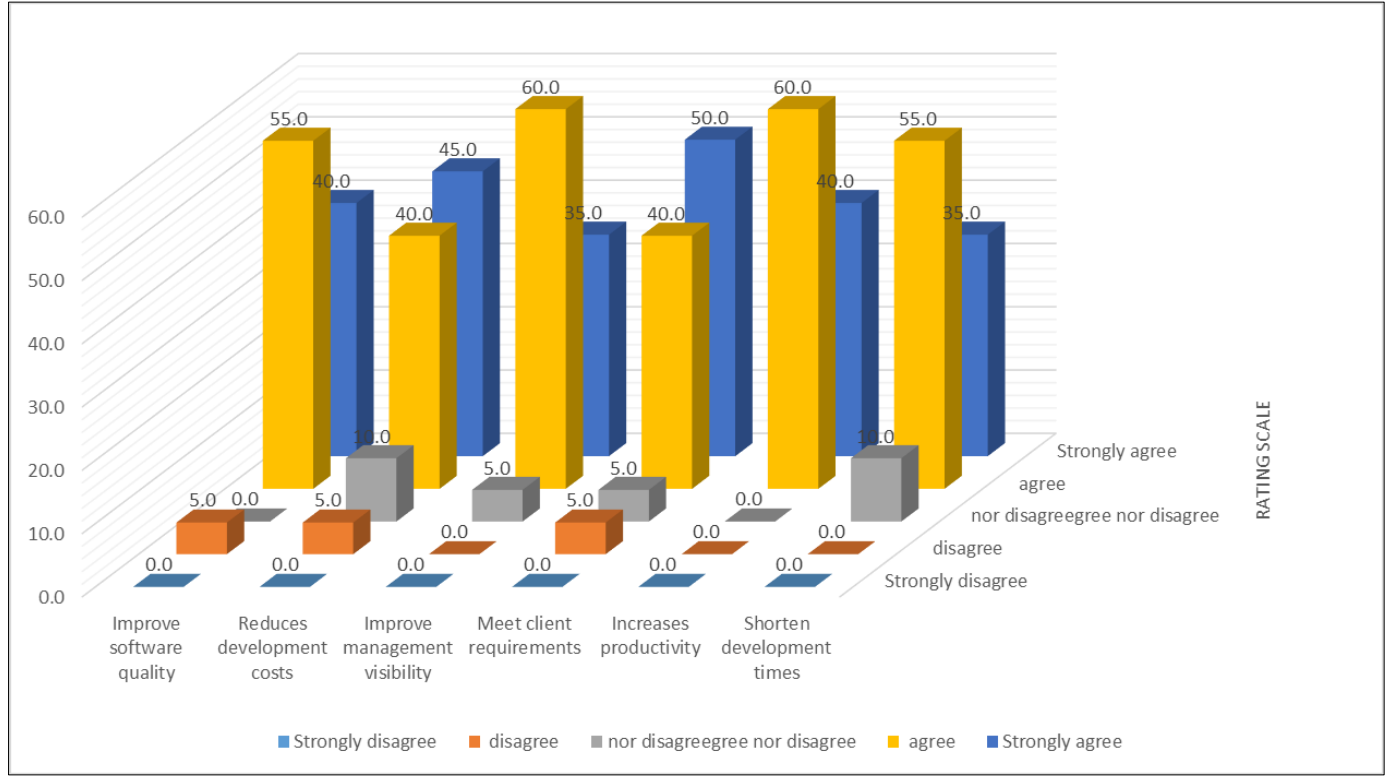

Fig. 1. Rating the benefits of using the SPI.

The results show that $60 \%$ of the respondents agree that the usage of SPI standard will increase productivity and improve management visibility; whereas, 55\% agree that the SPI standard will shorten development time and improve software quality. Moreover, the respondents strongly agree that the usage of SPI standard has a significant impact on meeting client requirements $50 \%$ and reducing development cost $45 \%$. These presented results are consistent with [34], in which they stated that, most software houses are not able to quantify the benefits of implementing SPI standard clearly.
Secondly, the reasons of not using the SPI standards were shown in Fig. 2. In this graph, the rating process shows that most of the respondents strongly agree that inexperienced staff $59.1 \%$ and staff turnover $54.4 \%$ are also reasons for not using of SPI. The most disappointing aspect about the reasons of not using of SPI standard throughout the ratings scales is that $(64 \%)$ of the respondents agree that the usage of SPI standard is time consuming, and $55 \%$ agree that there is a difficulty in understanding the SPI standard. However, this result occurred because $73 \%$ of the respondents are suffering from the lack of resources to adopt the SPI standard.

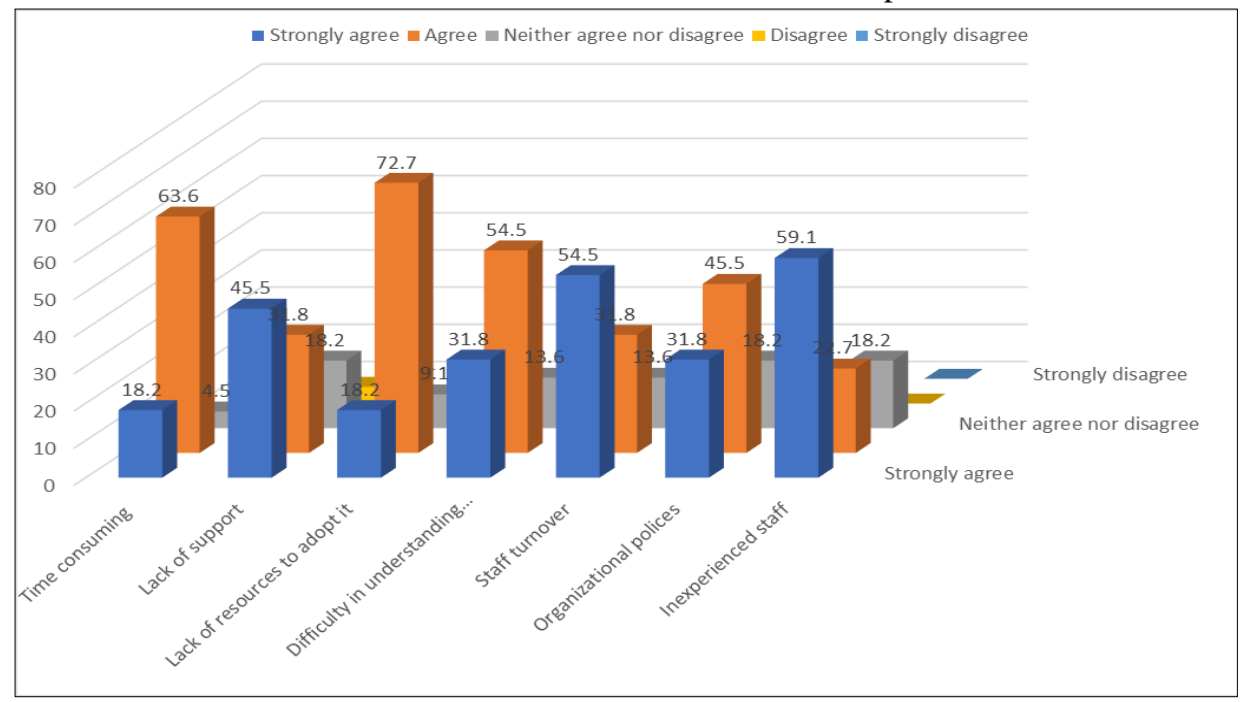

Fig. 2. Rating reasons of not using SPI. 


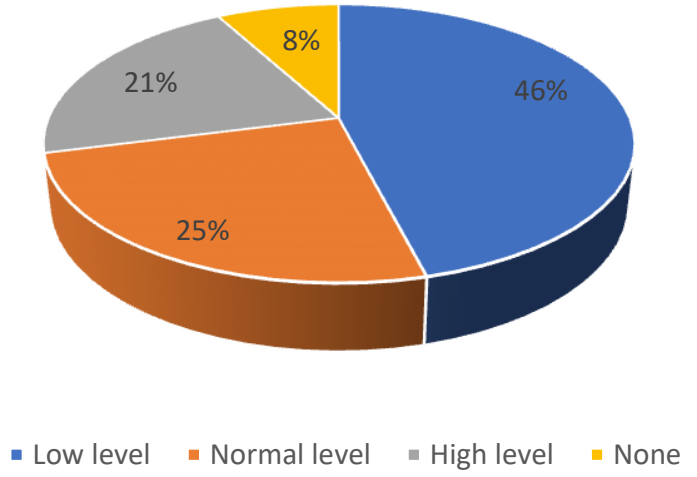

Fig. 3. Knowledge of SPI implementation.

The result of the level of knowledge in SPI practices is depicted in Fig. 3. Despite the importance of the SPI standard, majority of the employees $46 \%$ have low knowledge level of SPI practice and a $25 \%$ have normal level of practices. It is also shown that $8 \%$ of the respondents have zero knowledge while only $21 \%$ have high level of knowledge in SPI standard.

On the other hand, Fig. 4 shows the level of employees' experience in small and medium size Saudi Arabian companies. Slightly over two third $69 \%$ of the respondents have between 5 to 10 -years of experience and only a quarter of them $23 \%$ have more than 10 years of experience. However, this was an expected result since the SPI standards were not written for developed organizations with fewer than 25 employees and are consequently difficult to apply in such small settings.

In addition, it is shown in Fig. 5 that $46 \%$ of the respondents have used the standard ISO 9000 in software development and a very small percentage only $17 \%$ of the respondents used CMMI. Although, the ISO 9001 standard has been implemented by many organizations in 187 countries [35], yet, this standard is criticized in journals, textbooks and at conferences as it is written for industry (i.e large size), and its application to the development in the small size organizations may pose some problem [36]-[38].

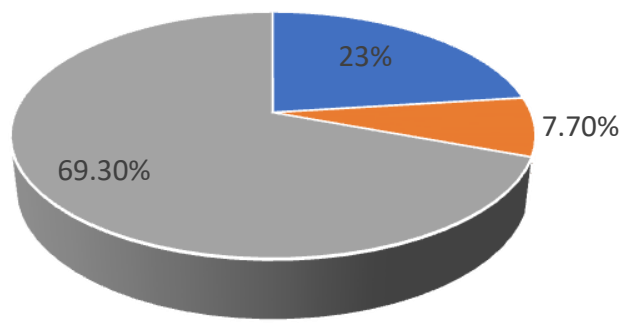

- (1-5) Years - (5-10) Years " >10 Years

Fig. 4. The level of employee's experiences.

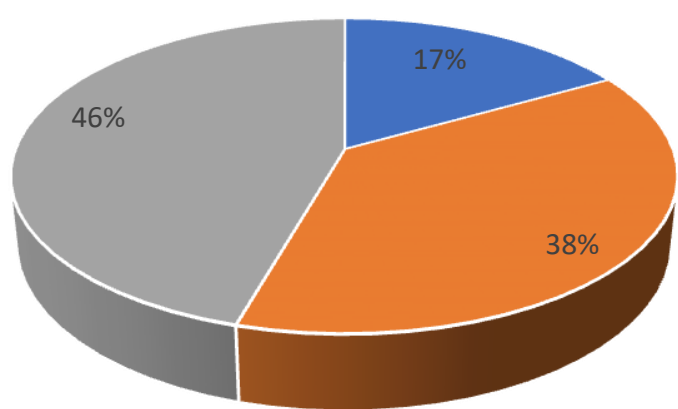

- CMMI $\because$ SPICE $=$ ISO9000

Fig. 5. SPI standard used in Saudi Arabia.

Regarding the duration of SPI adoption in the organization, the result of the survey shows that $62 \%$ of the respondents stated that they adopted process improvement program for more than 5 years. Meanwhile, $13 \%$ of the respondents are yet to start adopting the SPI standard as shown in Fig. 6.

More often, problems arise in every project. Therefore, the project managers have to strictly comply with software development process and with management tools such as budget management, resource allocation, time control, priority of tasks, testing technology and decision-making tools. Consequently, whether the projects are completed on time and (or) on budget is investigated. The participants were asked if they are receiving technical training courses or not and whether the communication method is formal or informal. As shown in Fig. 7, 62\% of the projects are completed on budget while $38 \%$ are completed over budget. Comparatively, the results stated that $46 \%$ of projects completed on time while $54 \%$ are completed over time.

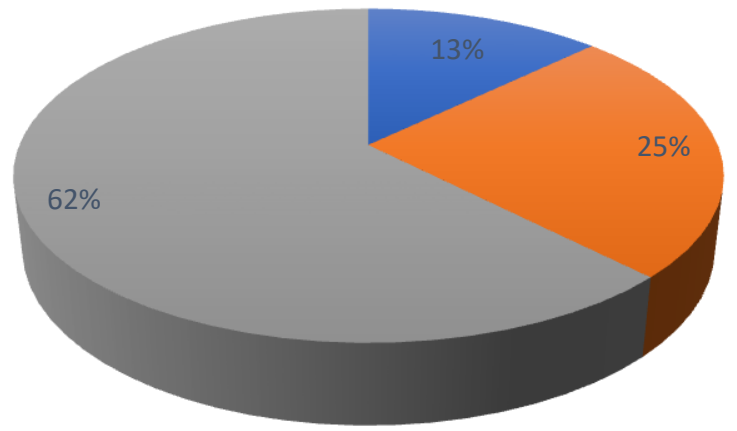

- No Adoption

(1-5) years of adoption

- 5 years of adoption

Fig. 6. Duration of SPI standard adoption. 


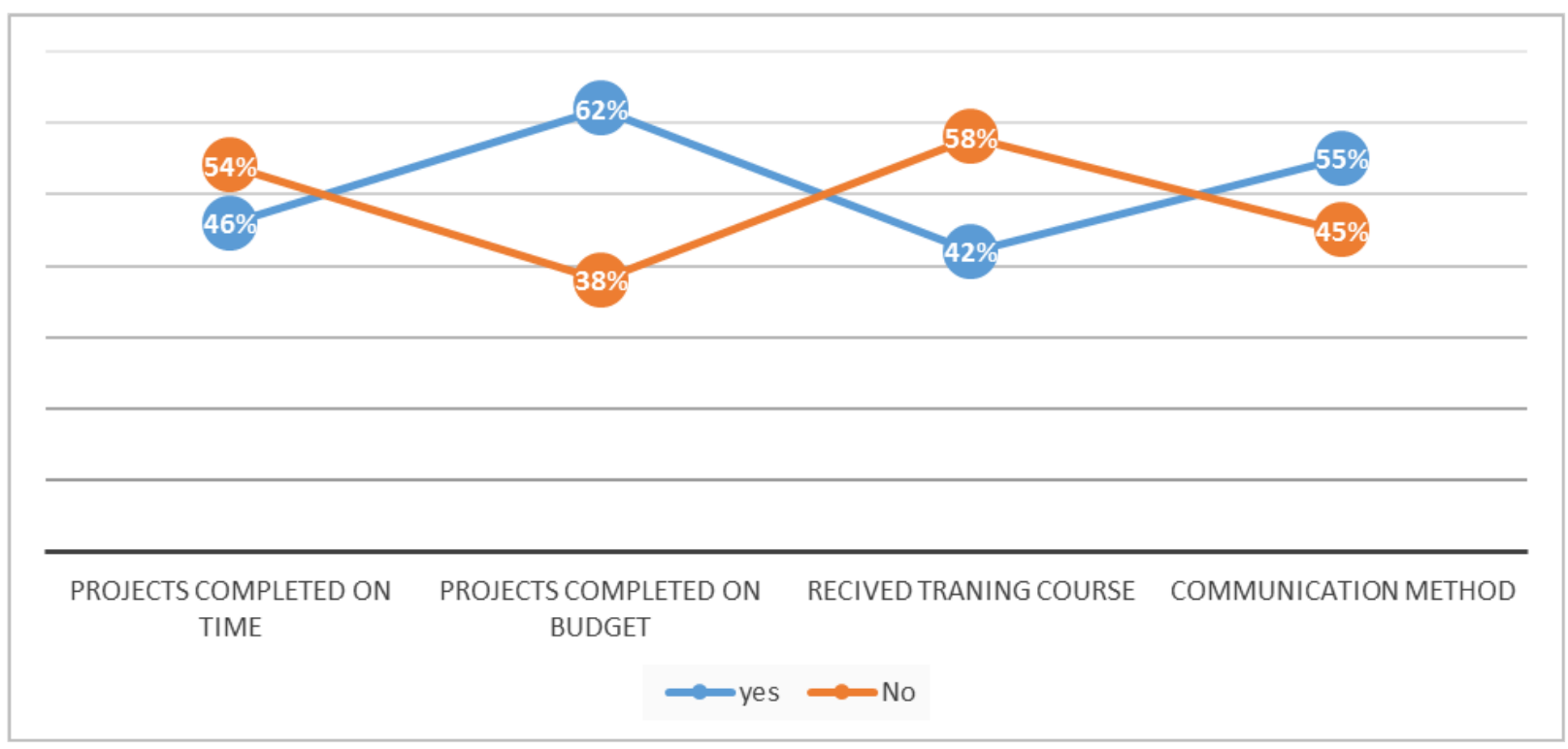

Fig. 7. Project management method.

The training courses provided to employees working in the Saudi SMEs are also surveyed. In this survey, $58 \%$ of the respondents stated that they did not receive training courses to improve their development skills while $42 \%$ of them indicated that they received course training to improve their managerial and technical skills. Given these point, self-training method represented $46 \%$, while $54 \%$ used formal training to improve their knowledge. Moreover, with reference to communication within the organizations, $55 \%$ of the respondents stated that they use the formal communication channels of the organization such as official letters and Emails. This means that the flow of information between sender and receiver is controlled. Often, Information is collected and flows up to the top levels of management for review and decision making, while orders flow down from the top to the place where it will be implemented. On the other hand, $45 \%$ of the respondents stated that the communication between employees is informal or through the ad-hoc method; in which the interchange of information does not follow any channels. Often, they use social media tools such WhatsApp, Tweeter and Telegram with any documentary evidence.

The final question outlined project management (Tools and Techniques) adopted to execute and monitor the engineering process to ensure conformance of quality as per organization's standards. Better use of project management tool help planning, organizing, and managing project resources and tasks. After analyzing the results, it was found that the most dominant software tool used by project managers in Saudi Arabia is scheduling tools. The result shows that $37.5 \%$ of the respondents use scheduling tools, while $25 \%$ use quality management tools, $20.8 \%$ use project management tools and 16.7\% use CASE tools shown in Fig. 8.

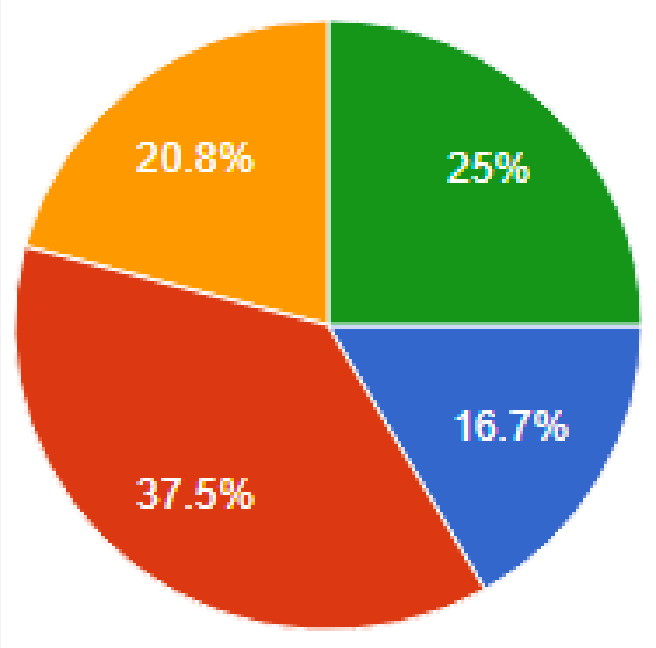

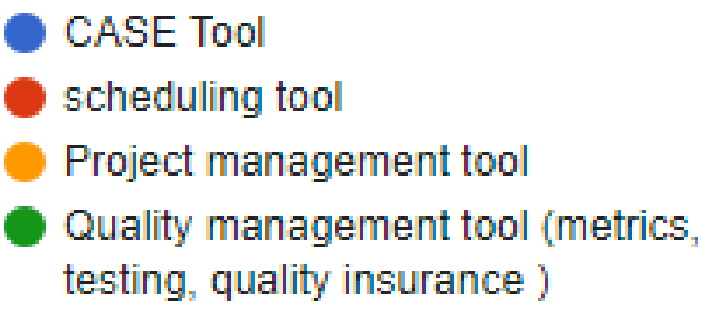

Fig. 8. Project management (tools and techniques). 


\section{DISCUSSION}

The results presented in Fig. 1 are interesting where respondents identified multiple benefits of using of SPI standard such as increased productivity and management visibility as well as shortening development time and improve quality. This was confirmed by [40] who claimed that SPI models and standards can improve the quality of software by reducing cost and increasing productivity. In addition, the respondents highlighted the significant impact of SPI on meeting clients' requirements and reducing development cost. This shows a good level of awareness among the respondents on the significance of SPI adoption. However, as shown in Fig. 2, most of the respondents indicated lack of resources as well as time consuming as the top reasons for not using SPI standard in Saudi Arabia. The same reasons were highlighted in other studies in different countries such as Canada [40] and English-Speaking Caribbean [41]. In addition, and with reference to the lack of resources, it is worth mentioning that SMEs have limited financial recourses which negatively impact resources needed to adopt SPI.

As for the level of knowledge, the majority of respondents have low level of knowledge as shown in the results in Fig. 3. This result is considered normal as per Colomo-Palacios [2] claim that the long goal in SPI is to accelerate the implementation and institutionalization of improved software development practices. The knowledge will be obtained individually by the participants in the process, and then expand the level of the organization to be applied in new projects.

Furthermore, it can be argued from the results presented in Fig. 7 about project management methods that Saudi's SMEs are majorly concerned with the delivery of software products to client on budget within the stipulated time and this is consistent with findings in [23]. It also means that, they do not pay any critical attention to best practice standards in software development but find it easier to employ ad-hoc and/or agile approach, enabling quicker delivery of a working software product. However, without properly defined software process and practices, it would be difficult to deliver software product on time and right on budget. On the other hand, and with reference to project management techniques, several tools are used by companies to conduct projects. This result is consistent with the view of Almobarak et al. [39], who have reported that more than $90 \%$ of respondents use at least one project management tool to help in planning, organizing, and managing project resources and tasks.

All in all, the current SPI adoption level in Saudi Arabian SMEs is very much in the low level. This has been shown in the result in the previous section as well as from the discussion presented here.

\section{CONCLUSION AND FUTURE WORK}

This study was conducted to investigate small and medium size organizations in Saudi Arabia on their utilization of SPI standards and to collect data to identify problems and potential solutions to help them apply these standards. Our research found that although there is positive favor towards SPI adoption among Saudi organizations, it seems that the practical understanding of SPI adoption is extremely yet to be mature amongst small and medium size software companies. A primary reason is that many staff do not have the appropriate knowledge to deal with the process of SPI and many managers are reluctant to implement SPI because of the associated costs. Most of staff in these organizations are aware that the adoption of SPI will increase productivity, improvement management, shorten development time and improve software quality. However, inexperienced staff and staff turnover are the main reasons for not using of SPI.

Some recommendations can be drawn here to improve the adoption of software engineering practice and techniques:

- Training: One important factor that influences the adoption of SPI is proper and regular education and training of technical staff.

- Finding organization weakness: The key issue in accelerating the adoption of SPI practices is to identify the areas that need most improvement and then find the best way to support organizations in improving these areas.

Common weaknesses are in need to be solved in workplaces in the majority of organizations. These working obstacles can be minimized by strengthening the skills and enriching the knowledge of the employees. Providing the necessary training will bridge the gap and reduce the weaknesses between the levels of the knowledge of the employees and provide them with a common base.

Since the study is performed in Saudi Arabia, it is not clear whether the result can be generalized in the countries that have similar characteristics especially other Arabic countries. Using this data in various environments and contexts to provide cross-cultural comparisons may enrich the literature and result in understanding and applying SPI techniques to achieve high quality process. Assuming that software is developing rapidly and significantly and SPI is becoming very importantly day by day, a future work can use this study as first step towards producing software process improvement standard for Saudi Arabian industry. Also future research can investigate the relationship between organizational size and the SPI success by identifying factors and barriers influencing the SPI implementation activities for large organizations.

\section{ACKNOWLEDGMENT}

The author would like to Thank Shaqra University, Saudi Arabia for providing a great research environment with excellent infrastructure. The author would also like to thank all participants who have completed the questionnaire.

\section{REFERENCES}

[1] Fauzi, S. S. M. (2011). Software Process Improvement and Management: Approaches and Tools for Practical Development: Approaches and Tools for Practical Development, IGI Global.

[2] Colomo-Palacios, R. (2014). Agile Estimation Techniques and Innovative Approaches to Software Process Improvement, IGI Global.

[3] SCAMPI Upgrade Team, "Standard CMMI Appraisal Method for Process Improvement (SCAMPI) A, Version 1.3: Method Definition Document," Softw. Eng. Inst., Mar. 2011.

[4] C. N. Murphy and J. Yates, The International Organization for Standardization (ISO): global governance through voluntary consensus. Routledge, 2009. 
[5] W. Melo, K. El Emam, and J. Drouin, "SPICE: The theory and practice of software process improvement and capability determination," IEEE Comput. Soc. Wiley Los Alamitos CA, 1998.

[6] K. E. Emam, W. Melo, and J.-N. Drouin, SPICE: The theory and practice of software process improvement and capability determination. IEEE Computer Society Press, 1997.

[7] B. Efron and R. J. Tibshirani, An introduction to the bootstrap. CRC press, 1994.

[8] B. Efron, "Bootstrap methods: another look at the jackknife," in Breakthroughs in Statistics, Springer, 1992, pp. 569-593.

[9] P. Pande, R. Neuman, and R. Cavanagh, The Six Sigma way team fieldbook: An implementation guide for process improvement teams. McGraw Hill Professional, 2001.

[10] R. G. Schroeder, K. Linderman, C. Liedtke, and A. S. Choo, "Six Sigma: Definition and underlying theory," J. Oper. Manag., vol. 26, no. 4, pp. 536-554, 2008.

[11] R. B. Coronado and J. Antony, "Critical success factors for the successful implementation of six sigma projects in organisations," TQM Mag., vol. 14, no. 2, pp. 92-99, 2002.

[12] I. Garcia, C. Pacheco, and J. Calvo-Manzano, "Using a web-based tool to define and implement software process improvement initiatives in a small industrial setting," IET Softw., vol. 4, no. 4, pp. 237-251, 2010.

[13] C. Y. Laporte, S. Alexandre, and A. Renault, "The application of international software engineering standards in very small enterprises," Softw. Qual. Prof. Mag., vol. 10, no. 3, 2008.

[14] M. Staples, M. Niazi, R. Jeffery, A. Abrahams, P. Byatt, and R. Murphy, "An exploratory study of why organizations do not adopt CMMI," J. Syst. Softw., vol. 80, no. 6, pp. 883-895, 2007.

[15] I. Richardson and C. G. Von Wangenheim, "Guest editors' introduction: Why are small software organizations different?," IEEE Softw., vol. 24, no. 1, pp. 18-22, 2007.

[16] C. Y. Laporte, S. Alexandre, and A. Renault, "The application of international software engineering standards in very small enterprises," Softw. Qual. Prof. Mag., vol. 10, no. 3, 2008.

[17] M. A. T. Almomani, S. Basri, A. K. B. Mahmood, and A. O. Bajeh, "Software Development Practices and Problems in Malaysian Small and Medium Software Enterprises: A Pilot Study," in IT Convergence and Security (ICITCS), 2015 5th International Conference on, 2015, pp. 1-5.

[18] H. Oktaba and A. Vázquez, "MoProSoftß: A Software Process Model," Softw. Process Improv. Small Medium Enterp. Tech. Case Stud. Tech. Case Stud., 2008.

[19] M. P. Thapliyal and P. Dwivedi, "Software process improvement in small and medium software organisations of India," Int. J. Comput. Appl., vol. 7, no. 12, pp. 37-39, 2010.

[20] S. Ibrahim and R. Z. R. M. Ali, "Study on acceptance of customised Software Process Improvement (SPI) model for Malaysia's SME," in Software Engineering (MySEC), 2011 5th Malaysian Conference in, 2011, pp. 25-30.

[21] A. R. Khan, R. Akbar, and D. W. H. Tan, "A Study on Global Software Development (GSD) and Software Development Process in Malaysian Software Companies," J. Telecommun. Electron. Comput. Eng. JTEC, vol. 8, no. 2, pp. 147-151, 2016.

[22] S. S. M. Fauzi and M. H. N. M. Nasir, "Software Process Improvement Models Implementation in Malaysia," Innov. Adv. Comput. Sci. Eng., pp. 85-90, 2010.

[23] R. Z. R. M. Ali and S. Ibrahim, "An application tool to support the implementation of integrated software process improvement for Malaysia's SME," in Software Engineering (MySEC), 2011 5th Malaysian Conference in, 2011, pp. 177-182.
[24] M. A. Cusumano and C. F. Kemerer, "A quantitative analysis of US and Japanese practice and performance in software development," Manag. Sci., vol. 36, no. 11, pp. 1384-1406, 1990.

[25] L. Groves, R. Nickson, G. Reeve, S. Reeves, and M. Utting, "A survey of software development practices in the New Zealand software industry," in Software Engineering Conference, 2000. Proceedings. 2000 Australian, 2000, pp. 189-201.

[26] I. Saastamoinen and M. Tukiainen, "Software process improvement in small and medium sized software enterprises in eastern Finland: A stateof-the-practice study," in European Conference on Software Process Improvement, 2004, pp. 69-78.

[27] S. N. M. Shah, M. Khalid, A. K. B. Mahmood, N. Haron, and M. Y. Javed, "Implementation of software process improvement in pakistan: An empirical study," in Computer \& Information Science (ICCIS), 2012 International Conference on, 2012, vol. 2, pp. 1006-1013.

[28] M. Niazi, "An exploratory study of software process improvement implementation risks," J. Softw. Evol. Process, vol. 24, no. 8, pp. 877894, 2012.

[29] M. Staples, M. Niazi, R. Jeffery, A. Abrahams, P. Byatt, and R. Murphy, "An exploratory study of why organizations do not adopt CMMI," J. Syst. Softw., vol. 80, no. 6, pp. 883-895, 2007.

[30] G. Coleman and R. O'Connor, "Using grounded theory to understand software process improvement: A study of Irish software product companies," Inf. Softw. Technol., vol. 49, no. 6, pp. 654-667, 2007.

[31] M. H. N. Nasir, R. Ahmad, and N. H. Hassan, "Issues in the implementation of software process improvement project in Malaysia," WSEAS Trans. Inf. Sci. Appl., vol. 5, no. 6, pp. 1031-1043, 2008.

[32] R. Asato, M. de M. Spinola, I. Costa, and W. H. de F. Silva, "Alignment between the business strategy and the software processes improvement: A roadmap for the implementation," in PICMET '09 - 2009 Portland International Conference on Management of Engineering Technology, 2009, pp. 1066-1071.

[33] M. A. Resources Information, Software Design and Development: Concepts, Methodologies, Tools, and Applications: Concepts, Methodologies, Tools, and Applications. IGI Global, 2013.

[34] "Software process improvement via ISO 9000: Results of two surveys among European software houses - IEEE Conference Publication." [Online]. Available: http://ieeexplore.ieee.org/document/495524/. [Accessed: 24-Oct-2017].

[35] B. Manders, H. J. de Vries, and K. Blind, "ISO 9001 and product innovation: A literature review and research framework," Technovation, vol. 48-49, no. Supplement C, pp. 41-55, Feb. 2016.

[36] D. Stelzer, W. Mellis, and G. Herzwurm, "A critical look at ISO 9000 for software quality management," Softw. Qual. J., vol. 6, no. 2, pp. 65-79, Jun. 1997.

[37] T. Stålhane and G. K. Hanssen, "The Application of ISO 9001 to Agile Software Development," in Product-Focused Software Process Improvement, 2008, pp. 371-385.

[38] G. Coleman and R. O'Connor, "Investigating software process in practice: A grounded theory perspective," J. Syst. Softw., vol. 81, no. 5, pp. 772-784, May 2008.

[39] N. AlMobarak, R. AlAbdulrahman, S. AlHarbi, and W. AlRashed, "The Use of Software Project Management Tools in Saudi Arabia: An Exploratory Survey," Int. J. Adv. Comput. Sci. Appl. IJACSA, vol. 4, no. 7, 2013.

[40] D. Chevers, "Software Process Improvement: Awareness, use, and Benefits in Canadian Software Development Firms" Revista de Administração de Empresas, 57(2), 2017, 170-177

[41] D. Chevers, "The Adoption of Software Process Improvement Programs in the English-Speaking Caribbean" in International Conference on Information Resources Management (CONF-IRM), 2014. 